\title{
Research on live line auto-testing technology for transmission line insulators
}

\author{
Tao GUO ${ }^{1, a}$, ,Wei WANG ${ }^{1, b}$, Heng YANG ${ }^{1}$ and Xin-xing YUAN ${ }^{2, c}$
}

${ }^{1}$ Guizhou power grid transmission operation maintenance branch, Guiyang, China,500003

\author{
${ }^{2}$ Wuhan Kedio Electric Power Technology Co.,Ltd, Wuhan, China,430074 \\ a79169957@qq.com, b525492887@qq.com, ${ }^{\mathrm{c}} 15827527678 @ 163 . c o m$
}

Keywords: Insulator; Automatic detection; Guide rail;

Abstract. insulator is an important part of transmission line, and its maintenance of is of great significance. Duo to The insufficiency of traditional way of insulator detection, this paper puts forward a kind of automatic on-line detection method. The sliding carriage which carries a detection device slides along the guide rail smoothly and precisely, the field data is measured reliably. Field practice shows that this technology can increase efficiency and reduce the labor intensity greatly.

\section{Introduction}

To solve the problem of faulty insulator detection, various detection methods were studied at home and abroad $[1,2,3]$. Such as voltage distribution method, corona pulse current method, ultrasonic method, infrared imaging method, ultraviolet imaging method, electric field measurement method, etc. Among them, the electric field distribution method detect internal insulation defects live line, and instruments used in this method is simple, low requirement to the external environment. Using electric field distribution method to detect insulator in principle and actual application fully meets the actual demand[4,5].

According to the above problem, this paper studies the electric field intensity measurement, automatic control and other advanced technology[6], develops a set of insulator on-line detection device, to ensure that the electric field detection device can slide along the insulator string on a guide rail smoothly and precisely, and by controlling the mechanical testing instruments, electric field data is accurately and reliably measured. By that way, it achieves the goal to detect faulty insulator, effectively reduce the labor intensity of operators.

\section{Faulty principle of insulator detection}

This faulty insulator detecting device with electric field method is the basic principle[7], its core is based on the head of the optical electric field sensor to measure electric field. Light sources into the single-mode fiber laser coupling, sensing head inside through coupling the laser collimating lens to the space, with the partial linear polarized makes it, after a quarter wave plate again divided into circularly polarized light, the fast axis parallel to the electro-optic crystal respectively under the effect of electric field of two induction spindle, through the crystal, the polarization is changeless, but phase changed, and is related to the measured field intensity. With partial detector two perpendicular polarized light beam to extract the same component, get interference light intensity. The emergent light for photoelectric conversion, the change of light intensity changes into electrical signals, reflects the change of the electric field to be tested.

In the actual test, the testing instrument slides along the surface of insulator, it can automatically record the electric field data and the serial number of insulator[8]. After completion of measurement, data is exported. According to the measured data curve, by comparison with the normal electric 
field distribution curve, determining whether it is faulty insulator, as shown in Fig.1.

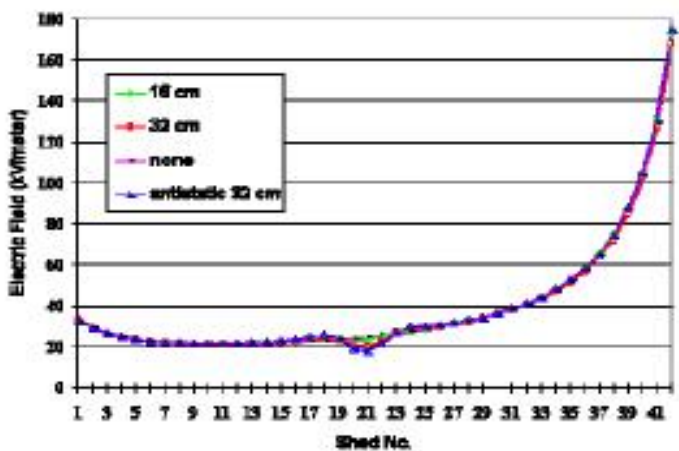

Fig.1. Normal electric field distribution curve and faulty situation

\section{Precise movement and positioning scheme}

In order to solve the problem of the moving of detection sensor can smoothly, and let the testing data without fluctuation and improve precision and stability of the electric field intensity measuring, it must ensure that carry the robot car's detection module is the precisely controlled and located.

The composition of the running mechanism. Movement mechanism of the detection device consists of drive mechanism, motion guide rail, and auxiliary safety, as shown in Fig.2.

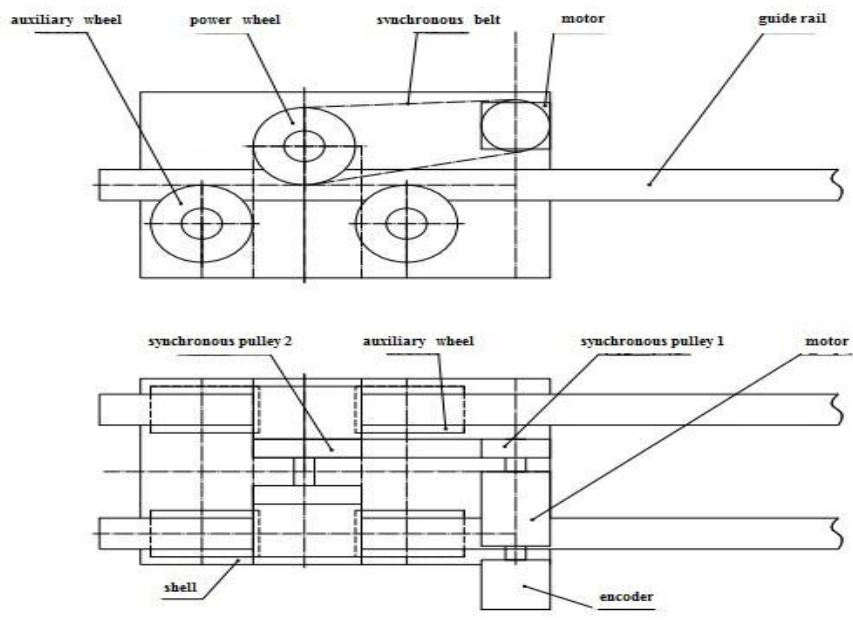

Fig.2 Move organization structure

1) Drive mechanism. Selection of belt machine of decelerate of planet of biaxial power brushless using DC motor output, motor and arc-gear synchronous belt pulley, synchronous belt for insulation materials, positioning is accurate, which ensures the smooth. Transmission is equipped with tensioning device, to ensure reliable transmission, at the same time, facilitate synchronous belt replacement. Momentum wheel by belt auxiliary ring grooves, bearing, synchronous belt wheel, the gear of a few parts, and adopts screw connection between shell. Auxiliary matrix for MC nylon, with arc grooves, adapt to the motion guide rail insulating rod body form, containing ceramic bearings and support shaft, rotating flexible. And momentum wheel clamping insulating rod body together, training wheels with adjustable spacing and momentum wheel rotation axis adjusting mechanism, can easily remove from the motion guide robot body.

2) Motion guide rail. Move guide is made of epoxy resin fiber center with T slots, can cooperate with the transmission rack, both ends have threaded connection plug, the hall element installation position, can restrict movement scope of robot ontology on the guide rail. Rack transmission matrix by $\mathrm{MC}$ nylon, section structure, composed of the whole rack cutting, splicing, cross section for $\mathrm{T}$ type, convenient to embed insulating rod body, every day there are at least one end of the $45^{\circ}$ 
inclined plane form, the back of the rack is equipped with elastic strip, ensure that rack tooth profile stitching. Rapid locking device by eccentric locking lever and special rail joint of two parts. When folding movement guide rail straighten, presses the eccentric wheel handle, lock the card into the buckle, can quickly complete guide rail length is longer. Don't need to lengthen, loosen the eccentric wheel handle, after folding rod body with card into the tail screens.

Between the two insulating rod bodies with a diameter of $6 \mathrm{~mm}$ double end studs, outsourcing insulation rubber layer, keep the spacing between the two poles, strengthening of the rod body rigidity.

3) Auxiliary safety. Auxiliary fixed bracket, designed to reduce the labor intensity of operators. Using activity articulated in the form of architecture, with a certain Angle range Vernier function, triangle fixed, solid structure, can be threaded jacking method, convenient fixed in tower Angle steel frame.

Failure, in order to ensure the driving mechanism can be safely and timely recovery robot ontology, in the shell and supporting wheel clamping device install insulation rope, convenient power loss, loosen the auxiliary wheel clamping device, using another security insulation rope, ontology back safely.

Research of Precise positioning technology.1) Photoelectric encoder. This article will use the encoder to follow a moving car wheel shaft encoder design of synchronous rotation, the number of pulses through the records in the process of the movement, to accurately control the movement of the car distance. By adopting the combination of calculation and measurement range in the form of a circle running distance can be calculated by type 1 .

$$
\mathrm{C}=\pi \mathrm{MZ}
$$

$\mathrm{C}$ is the distance of the gear rotates; $\mathrm{M}$ is the module of gear; $\mathrm{Z}$ is the gear teeth.

Through calculation of the software can convert circular motion to linear distance:

$$
\mathrm{S}=\mathrm{nC}
$$

If motor flipping produced $-\Delta \mathrm{n}$, we can determine the location of its detection terminal by Eq. 3 :

$$
\mathrm{S}=(\mathrm{n}-\Delta \mathrm{n}) \mathrm{C}
$$

2) Hall element. In this design, the insulator of the hall element close to the installation of ontology in the guide rail at the ends of the magnets, magnetic field changes. Flip the transistor output level, trigger the CPU interrupt, the CPU can control motor stop rotating, To prevent the device out of range and resistance fault.

Way of working. The rail is adjusted to be greater than the length of the insulator strings measurement requirements, the power drive unit is mounted on the robot body on the motion guide, locking the auxiliary wheel locking handle, making secondary slots auxiliary wheel and wheel clamping force on the wheel rails, ensuring stable robot movement on the track, not fall, will be mounted on the auxiliary rail mounting bracket. Start the motor, relying belt transmits power to the wheel with the gear part of the power, rack rails mounted on the drive by the housing forward and backward. Encoder and motor through the coupling connection with the operation of the Hall element at the same time, to determine the specific location of the car travels. After the completion of testing, backtrack. If the mechanical failure, the use of insulated Rope slack open auxiliary wheel locking handle, after losing clamping force can be easily towed by an insulating rope.

\section{Design Control and communication module}

Control circuit of the main role is to control the action of testing equipment, as well as the subsequent information processing, mainly including start detection device, control the movement of the actuator, receive insulator electric field testing data, packaging required data detection, 
location, time, etc., and related parts. Controller hardware structure function as shown in the figure below:

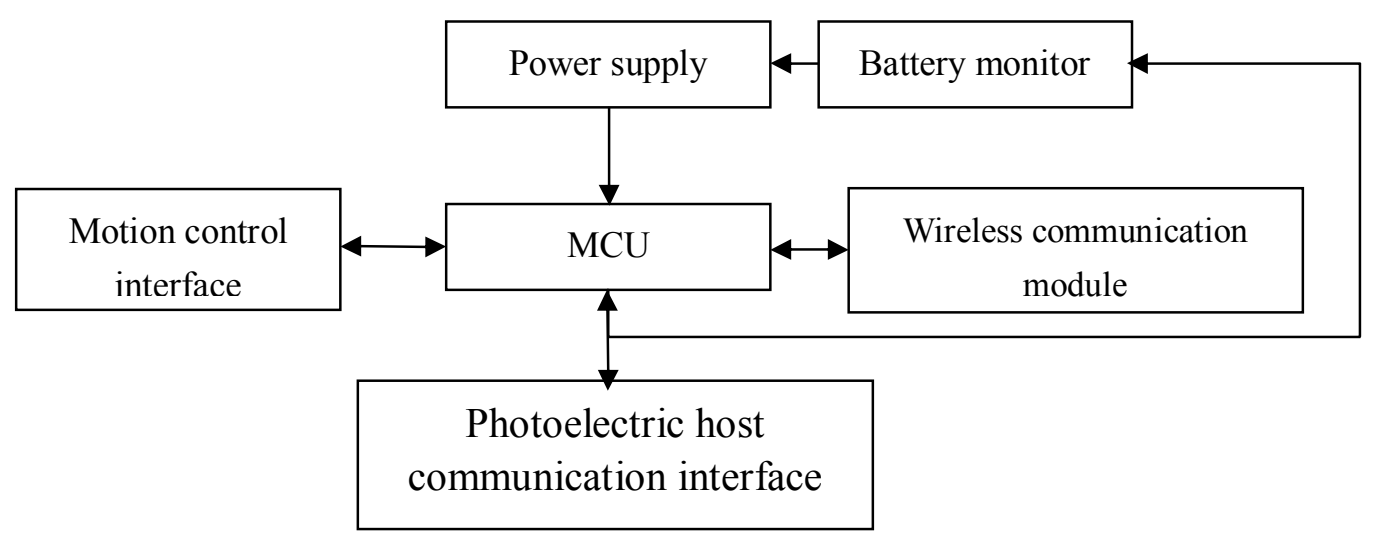

Fig.3 Functional Diagram

Communication control section and the main body of the robot using wireless transmission, convenient on-site communications and operations staff; insulator strings will be measured to detect data transmission to the next tower computers and remote control for field staff to get real-time observation Inspection results; income data can be stored and played back in real time, so you can easily check the entire tower insulator string data; real-time comparison of normal insulator string electric field distribution curves were analyzed to determine the position of the low value of zero insulator and displayed in real time to determine the results.

\section{Summary}

This paper introduces a kind of electric automation testing method for transmission line insulator. Optical electric field sensor is core element. Ontology along a guide rail movement by remote control detection, to test electric field of transmission line insulator smoothly and automatic, at the same time, it improves the detection accuracy greatly and reduces the work intensity.

\section{References}

[1] LI C.X, NIU B. , ZENG R., et al. Detection of Internal Insulation Defects of Composite Insulator Based on Electro-optic Electric Field Sensor [J]. High Voltage Engineering, 40(8), (2014).

[2] CHEN T., HE W., LIU X.M., XIONG D.. An On-line Ultraviolet Detecting System of EHV Transmission Lines. Automation of electric power systems, 29(7): 88-92(2005).

[3] CAO Tao, SUN D.Q, ZHAO D.L, et al. Research on the Application of Disc Type Porcelain Insulators Detection Robot in Overhead Transmission Lines. Insulators and Surge Arresters, 2: 004(2013).

[4] ZHU Hu, LI W.G, LIN Z.. Present and Future Development of Detection Methods for Composite Insulator. Insulators and Surge Arresters, (6): 13-17(2006).

[5] HUANG J.K., ZENG H.R., YANG J.P.. Application of Infrared Thermal Imaging Technology to Detection of Low and Zero Resistance Insulators. Insulators and Surge Arresters, 2: 010(2013).

[6] YANG Yi, CHEN Y.F., HONG Y.P., et al. Ultrasonic inspection of composite insulator. Nondesteructive Testing, 25(7): 337-339, 357(2003).

[7] Kontargyri V T., Plati L N., Gonos I F.et al. Measurement and simulation of the voltage distribution and the electric field on a glass insulator string. Science Direct, Measurement, 2008(41): 471-480. 
[8] ZHOU J.G., DENG Z.C., MAO S.C.: The Application of Insulator On-line Monitor System Based on Leakage Current. Northeast Electric Power Technology, 26(1): 16-18,37. (2005). 